\title{
Presence of specific T cell response after SARS-CoV-2 vaccination in rheumatoid arthritis patients receiving rituximab
}

\author{
Maurizio Benucci ${ }^{1}\left[\right.$ (1) Arianna Damiani ${ }^{2} \cdot$ Maria Infantino $^{3} \cdot$ Mariangela Manfredi $^{3} \cdot$ Valentina Grossi $^{3} \cdot$ Barbara Lari $^{3}$. \\ Francesca Li Gobbi ${ }^{1}$. Piercarlo Sarzi-Puttini ${ }^{4}$
}

Received: 1 July 2021 / Accepted: 8 July 2021 / Published online: 29 July 2021

(c) The Author(s), under exclusive licence to Springer Science+Business Media, LLC, part of Springer Nature 2021

\section{Dear Editor,}

A series of observations have shown that vaccination against SARS-CoV-2 in patients with immune-mediated diseases treated with rituximab is followed by the absence of production of neutralizing antibodies to RBD [1]. A previous study in 126 patients focused on the role of rituximab in vaccination against SARS-CoV-2 [2]. Another recent study shows that only patients who had repopulated for B lymphocytes exhibited an immune response to the vaccine against SARS-CoV-2. In the study, 11 patients repopulated but only 7 responded [3].

The aim of our study was to evaluate the humoral and cellular immune response to two doses of COVID-19 vaccine BNT162b2 mRNA in a series of patients with rheumatoid arthritis treated with rituximab.

We evaluated a group of patients with rheumatoid arthritis who had received the last infusion of rituximab 6 months earlier (group A 4 patients), a group of patients who had received the last dose of rituximab 9 months earlier (group B 5 patients) and a group of patients who had received rituximab 12 months earlier (group C 5 patients). All patients received two doses of BNT162b2 mRNA COVID-19 vaccine 21 days apart. Patients underwent evaluation of the

Maurizio Benucci

maubenucci@tiscali.it;

maurizio.benucci@uslcentro.toscana.it

1 Rheumatology Unit, S. Giovanni Di Dio Hospital, Azienda Sanitaria USL-Toscana Centro, Hospital S. Giovanni Di Dio, Via Torregalli 3, 50143 Florence, Italy

2 Rheumatology Unit, Department of Clinical and Experimental Medicine, University of Florence, Florence, Italy

3 Immunology and Allergology Laboratory, S. Giovanni Di Dio Hospital, Azienda USL-Toscana Centro, Florence, Italy

4 Rheumatology Unit, ASST-Fatebenefratelli L. Sacco University Hospital, University of Milan, Milan, Italy lymphocyte subpopulations with determinations of the B lymphocyte population (CD27 - naive, CD27 + memory, $\mathrm{CD} 38+, \mathrm{CD} 20+, \mathrm{CD} 19+$ ) evaluated by flow cytometry (FACS CANTO II, BD Biosciences), before the vaccination and 3 weeks after the second dose of vaccine. The value of anti-SARS-CoV-2 Spike-RBD IgG antibodies (IgG antibodies against S1-RBD protein) quantified by FEIA (ThermoFisher, Uppsala Sweden) was determined 3 weeks after the second dose of vaccine. In addition, SARS-CoV-2-specific $\mathrm{T}$ cell responses were determined by incubating isolated T cells with a SARS-CoV-2-specific peptide mix (a peptide mix of the SARS-CoV-2 spike protein) and measuring the release of interferon $\gamma$ by activated T cells using an ELISA system (IFN- $\gamma$ release assay, IGRA) according to the protocol of the manufacturer (SARS-CoV-2-IGRA, Euroimmun, Lubeck, Germany). All patients were in clinical remission at the time of vaccination and discontinued methotrexate in the week of the first and second vaccine administration according to the published recommendations $[4,5]$.

Table 1 shows the characteristics of the 14 patients. Four of the 14 patients had no or low values of anti S1-RBD antibodies. The evaluation of IFN- $\gamma$ production by the IGRA test showed in the 4 patients a mediated CD8 $+\mathrm{T}$ cell response with a value of $>2500 \mathrm{mU} / \mathrm{mL}$.

Literature data have shown a correlation between antibody response and circulating levels of CD19+ B lymphocytes after vaccination against SARS-CoV-2 in patients with immune-mediated diseases. However, we can observe a T cell mediated immune response even in patients with B cell depletion. This has recently been observed also by other authors [6]. It is not yet clear what level of immunogenicity is representative of vaccine efficacy. We do not know which extent of $\mathrm{T}$ cell response and for how long it is adequate to protect patients against virus infection after vaccination, but preliminary studies are promising. Our data also indicate that treatment with RTX may not preclude SARS-CoV-2 


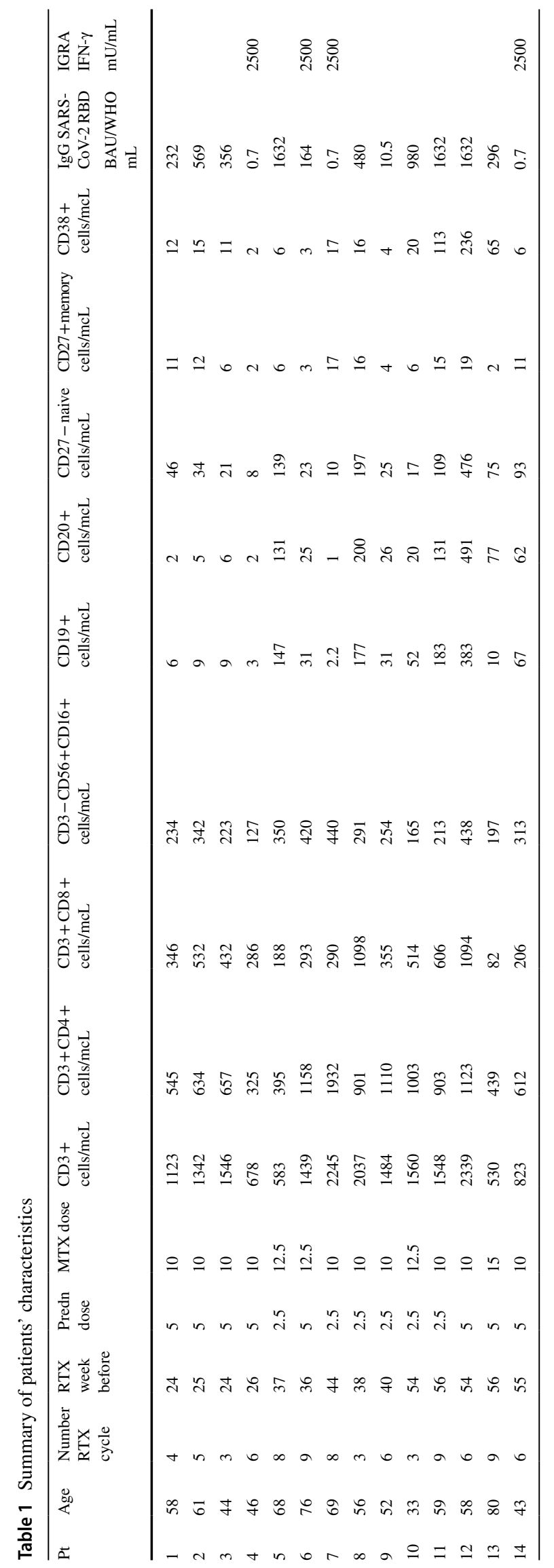


vaccination, as a cellular immune response will be activated even in the absence of circulating B lymphocytes.

\section{Declarations}

Conflict of interest The authors declare that they have no conflict of interest.

\section{References}

1. Connolly CM, Boyarsky BJ, Ruddy JA, Werbel WA, ChristopherStine L, Garonzik-Wang JM, et al. Absence of humoral response after two-dose SARS-CoV-2 messenger RNA vaccination in patients with rheumatic and musculoskeletal diseases: a case series. Ann Intern Med. 2021;M21-1451.

2. Boyarsky BJ, Ruddy JA, Connolly CM, Ou MT, Werbel WA, Garonzik-Wang JM, et al. Antibody response to a single dose of SARS-CoV-2 mRNA vaccine in patients with rheumatic and musculoskeletal diseases. Ann Rheum Dis. 2021; annrheumdis-2021-220289.

3. Spiera R, Jinich S, Jannat-Khah D. Rituximab, but not other antirheumatic therapies, is associated with impaired serological response to SARS- CoV-2 vaccination in patients with rheumatic diseases. Ann Rheum Dis. 2021; annrheumdis-2021-220604.

4. Bijlsma JW. EULAR December 2020 View points on SARSCoV-2 vaccination in patients with RMDs. Ann Rheum Dis. 2021;80(4):411-2.

5. Curtis JR, Johnson SR, Anthony DD, Arasaratnam RJ, Baden LR, Bass AR, et al. American College of Rheumatology guidance for COVID-19 vaccination in patients with rheumatic and musculoskeletal diseases-version 1. Arthritis Rheumatol. 2021;73(7):1093-107.

6. Bonelli MM, Mrak D, Perkmann T, Haslacher H, Aletaha D. SARS-CoV-2 vaccination in rituximab-treated patients: evidence for impaired humoral but inducible cellular immune response. Ann Rheum Dis. 2021; annrheumdis-2021-220408.

Publisher's note Springer Nature remains neutral with regard to jurisdictional claims in published maps and institutional affiliations. 